\title{
Über Papaveraldoxim
}

von

\author{
Dr. Robert Hirsch.
}

Aus dem chemischen Laboratorium der k. k. deutschen Universität in Prag.

(Vorgelegt in der Sitzung am 10. October 1895.)

In der Literatur finden sich bisher von Ketonen der Pyridinreihe das $\alpha$-Phenylpyridylketon von Skraup und Coben $z 1,{ }^{1}$ die Papaverinsäure und die Pyropapaverinsäure von Goldschmiedt, ${ }^{2}$ das $\beta$-Phenylpyridylketon von $B$ ernthsen und Wettegang, ${ }^{3}$ die von Engler ${ }^{4}$ dargestellten $\alpha$ - und $\beta$-MethylÄthyl-, Propyl-, Pyridylketone, ferner von Chinolinketonen ein Methyl- und ein Äthyl-Oxychinolinketon von Friedländer und Göhring, ${ }^{5}$ das Acetonylchinolin von Emil Fischer und Hans Kuzel, ${ }^{6}$ das Phenylchinaldinketon ron Geigy und Königs ${ }^{7}$ und das von Friedländer und Eliasberg ${ }^{8}$ gewonnene Methylacetylchinolin.

Der einzige Repräsentant eines Isochinolinketons ist bis jetzt das von Prof. G. Goldschmiedt ${ }^{9}$ gelegentlich seiner Untersuchungen über Papaverin dargestellte Papaveraldin.

1 Monatshefte für Chemie, 1883, 4.79.

2 Monatshefte für Chemie, $1885,381,394$.

3 Berl. Ber., XX, 1209.

4. Ebenda, XXIV, 2525.

5 Ebenda, XVI, 1838.

6 Ebenda, XVI, 163.

i Ebenda, XVIII, 2406.

8 Inaugural-Dissertation, 1890.

Monatshefte für Chemie, $1885,954$. 
Von manchen dieser Ketone sind Oxime zwar dargestellt und beschrieben worden, doch liegt über keines der Ketoxime eine Beobachtung bezüglich der zu erwartenden stereochemischen Isomeren vor.

Ich habe es daher auf Veranlassung des Herrn Prof. Dr. Goldschmiedt unternommen, das Papaveraldoxim in dieser Richtung zu untersuchen.

Bevor ich auf die Besprechung der gefundenen Thatsachen näher eingehe, sei hier erwähnt, dass, wenn ich schon heute das Ergebniss der bisherigen, durchaus noch nicht abgeschlossenen Untersuchungen veröffentliche, es mit Rücksicht auf den Umstand geschieht, dass ich diese Arbeit wegen meines Abganges vom Institute leider nicht fortsetzen kann. Dieselbe wird von anderer Seite fortgeführt werden.

Als gemischt aromatisches Keton, welchem nach Prof. Goldschmiedt folgende Formel zukommt:

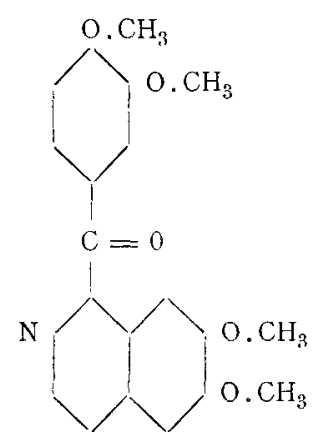

enthält das Papaveraldin einen stickstoffhältigen - den Dimethoxylisochinolin- - und einen Veratrolrest.

Das von Prof. Goldschmiedt ${ }^{1}$ dargestellte Oxim bildete flache Nadeln und schmolz bei $245^{\circ}$.

Ich erhielt nach dem angegebenen Verfahren, nach welchem man $70 \%$ Oxim erhält, als direct gewonnenes Rohproduct, bei einigen Darstellungen, ein Oxim rom Schmelzpunkt $235^{\circ}$, bei einer anderen Bereitung solches, welches bei $245^{\circ}$ schmolz, unter dem Mikroskope homogen erschien und entsprechend der

1 Monatshefte für Chemie, 1886, 489. 
Angabe Goldschmiedt's aus flachen Nadeln bestand. Die Präparate vom Schmelzpunkt $235^{\circ}$ erwiesen sich unter dem Mikroskope als ein Gemenge von äusserst feinen Nadeln und breiten, flachen Prismen. Eisenchlorid färbte die Lösungen beider Präparate gelbroth.

Durch wiederholtes Umkrystallisiren (5-6 mal) aus absolutem Alkohol gelang es, den Schmelzpunkt aller Präparate bis auf $254^{\circ} \mathrm{zu}$ erhöhen, die nun nur aus grossen, flachen Prismen bestanden. Um zu entscheiden, ob diese durch Umkrystallisiren erzielte Erhöhung des Schmelzpunktes nur eine Folge der Reinigung des Präparates war, oder ob hiedurch eine Umlagerung der labilen in die stabile Form des Ketoxims veranlasst worden war, wurden folgende Versuche mit Oxim vom Schmelzpunkt $235^{\circ}$ ausgeführt.

1. Das Oxim wurde mit der dreifachen Menge absoluten Alkohols im geschlossenen Rohre 3 Stunden auf $170^{\circ}$ erhitzt.

2. Das Oxim wurde bei gewöhnlicher Temperatur in Beckmann'scher Mischung gelöst, unter Kühlung trockenes Salzsäuregas eingeleitet und mehrere Tage bei gewöhnlicher Temperatur stehen gelassen.

3. Das Oxim wurde durch Einwirkung von Hydroxylaminchlorhydrat auf das Keton, ohne Zusatz von Natriumcarbonat, dargestellt.

Die Resultate sind folgende:

Bei dem Versuche 1 wurde der Schmelzpunkt des Oxims durch das Erhitzen nicht geändert.

Versuch 2 ergab nach 24 Stunden zunächst gelbe Krystalle einer Salzsäureverbindung, die abfiltrirt, zwischen Filtrirpapier getrocknet und in Wasser gelöst, mit Natriumcarbonat zerlegt wurden. Das so gewonnene Oxim hatte, ohne umkrystallisirt zu werden, den Schmelzpunkt $254^{\circ}$.

Versuch 3 lieferte nach mehrstündigem Kochen des in absolutem Alkohol gelösten Papaveraldins mit Hydroxylaminchlorhydrat, wobei die Reactionsflüssigkeit eine braune Färbung annahm, erst nachdem die Hälfte Alkohol abdestillirt worden war, braun gefärbte Krystalle von Oximchlorhydrat, die nach einmaligem Umkrystallisiren nur noch schwach gelblich gefärbt waren. 
Bei der Behandlung mit Wasser dissociirt die Verbindung theilweise und das abgeschiedene Oxim schmilzt bei $232^{\circ}$ bis $235^{\circ}$; zerlegt man hingegen das in verdünnter Salzsäure gelöste Oximchlorhydrat mit kohlensaurem Natron, so zeigt das so gewonnene Oxim den Schmelzpunkt von $253-254^{\circ}$. Das Oxim vom Schmelzpunkte $232-235^{\circ}$ konnte nach achtmaligem Umkrystallisiren auch in ein solches, das bei $254^{\circ} \mathrm{schmilzt}$, verwandelt werden.

Bekanntlich liefern nach Hantzsch ${ }^{1}$ die stereoisomeren Oxime verschiedene Chlorhydrate, aus denen man je nach der Behandlung mit Wasser, also in saurer Lösung oder mit Alkali, die beiden Isomeren getrennt erhalten kann. Es scheint also die eine Configuration in saurer, die andere in alkalischer Flüssigkeit begünstigt zu sein.

Es ist daher die Vermuthung gerechtfertigt, dass die verschiedenen Schmelzpunkte den beiden stereoisomeren Formen des Oxims zukommen, wobei das niedrig schmelzende labile durch die besprochenen Methoden in das höher schmelzende Oxim umgewandelt wird.

\section{Chlorhydrate der Papaveraldoxime.}

Wenn man Papaveraldoxim, gleichviel von welchem Schmelzpunkte, unter Erwärmen am Wasserbade in wässeriger Salzsäure auflöst, so krystallisiren nach dem Erkalten, je nach den Bedingungen, wie Mengenverhältniss des Oxims zur lösenden Salzsäure, Dauer des Erhitzens der Lösung, Temperatur, bei welcher die Verbindung ausfällt, intensiv gelbe oder weisse Chlorhydrate von in Bezug auf den Salzsäure- und Wassergehalt, je nach Umständen, wechselnder Zusammensetzung aus.

Der Umstand, dass die Chlorhydrate an der Luft Salzsäure und Wasser verlieren, so dass sie, nur zwischen Filtrirpapier getrocknet, zur Analyse verwendet werden mussten, bringt es mit sich, dass gut übereinstimmende Werthe bei den zahlreichen Analysen wasserhältiger Substanzen, die ich ausgeführt habe, nicht erhalten werden konnten; hingegen lieferten die bei

1 Berl. Ber. 1891, B. XXIV, 15. 
$110^{\circ}$ bis zur Gewichtsconstanz getrockneten gelben und weissen Rückstände untereinander und mit der Theorie gut übereinstimmende Zahlen.

Die verschiedenen Papaveraldoximchlorhydrate wurden aus Oxim sowohl mit 1-procentiger, als auch mit 7 - und 30-procentiger Salzsäure dargestellt. Die Stärke der Salzsäure übte keinen Einfluss auf den Wasser- und Salzsäuregehalt aus. Das Oxim wurde am Wasserbade nur so lange mit Salzsäure erwärmt, als zur Lösung nothwendig war; nach dem Erkalten schied sich dann das Chlorhydrat krystallinisch ab. Die Lösung ist unter allen Umständen goldgelb gefärbt. Die zahlreichen Versuche ergaben, dass sich stets aus concentrirten Lösungen (d. i. 1 Oxim : 10 Säure) unabhängig von der Concentration der Salzsäure beim Abkühlen ein hell gefärbter Syrup ausschied und gleichzeitig gelbes Chlorhydrat in feinen, seidenglänzenden, flachen Nadeln $(A)$, concentrisch zu Büscheln angehäuft, krystallisirte, die sich am Filter zu einem dichten Filz zusammenlegten. Der Syrup wurde beim Verreiben mit den Krystallen ebenfalls krystallinisch, so dass die ganze Flüssigkeit zu einem Krystallbrei erstarrte. Dagegen krystallisirt aus Lösungen von geringerer als oben angegebener Concentration gewöhnlich weisses Chlorhydrat aus; nur einmal war über Nacht aus einer verdünnten Lösung auch die gelbe Modification ausgefallen, was wohl mit der langsamen und ohne Störung vor sich gegangenen Abkühlung zu erklären war. Unter dem Mikroskope erschienen die Krystalle des weissen Chlorhydrates $(B)$ als rhombische, gut ausgebildete Prismen, die sich zu körnigen Aggregaten zusammenlagern.

Erhitzt man jedoch die Lösung des Oxims in Salzsäure längere Zeit am Wasserbade in einer Schale, so dass ein Theil des Lösungsmittels verdampft, so färbt sich die Lösung dunkler, die Krystallisation beginnt erst einige Stunden nach dem Erkalten und die gelben Krystalle sind wesentlich verschieden von den oben beschriebenen. Sie bilden grosse, quadratische Tafeln, die sich fest an die Wandung der Schale anlegen $(C)$.

Bei dem Versuche, die Lösung von Oxim in Salzsaure ohne Verdampfung von Lösungsmittel, also am Rückfluss- 
kühler längere Zeit zu kochen, wurden beim Erkalten gelbe Krystalle $(D)$ von ähnlicher Beschaffenheit, wie die erst beschriebenen $(A)$ erhalten. Doch zeigte sich, wie später mitgetheilt werden soll, bezüglich derselben eine Verschiedenheit mit jenen, in Bezug auf den Wassergehalt.

Genaue und oft wiederholte Beobachtungen haben ergeben, dass sich stets aus concentrirten Lösungen, in denen sich die Verbindung theilweise syrupös ausscheidet, neben der Hauptmenge der gelben, nadelförmigen Modification $(A)$ eine ganz geringe Menge weisser Krystalle ausschieden, die Anlass zu folgender interessanten Erscheinung gaben.

Während des Pressens dieses im Wesentlichen aus gelben Krystallen $A$ bestehenden Gemisches zwischen Filtrirpapier verschwindet in dem Grade, als die Verbindung trocken wird, die gelbe Farbe und das Präparat wird weiss. Wird nun das weiss gewordene Präparat neuerlich in der Mutterlauge gelöst und krystallisiren gelassen, so fallen wieder überwiegend gelbe Krystalle aus, mit welchen das Experiment wiederholt werden kann. Es ist nachgewiesen worden, dass nur dann die gelbe Modification als solche erhalten bleibt, wenn jede Spur des weissen Chlorhydrates ausgeschlossen wird, was in einzelnen Fällen dadurch erreicht werden konnte, dass die gelben Krystalle rasch abfiltrirt wurden, nachdem die in der Regel auf der Oberfläche schwimmenden weissen Kryställchen entfernt worden waren. Doch selbst in diesen Fällen geht die trockene gelbe Verbindung $(A)$ nach einigen Tagen unter Erhaltung der Krystallform, wenn auch unvollständig in die weisse $(B)$ über. Werden die gelben Krystalle in der Mutterlauge mit einem Glasstabe kräftig durchgerührt, so wandelt sich die gelbe Modification $(A)$ fast augenblicklich in die weisse $(B)$ um, eine Umwandlung, die nach einigen Tagen auch von selbst eintritt.

Anders verhält sich die gelbe tafelförmige Verbindung $(C)$, der keine weissen Krystalle beigemengt sind. Weder durch Reiben mit einem Glasstabe, noch durch längeres Stehen in der Mutterlauge wird der Übergang in die weisse Modification herbeigeführt.

Einmal im Besitze der beiden Chlorhydrate $A$ und $B$, braucht man bloss eine Lösung des Oxims in Salzsäure mit der einen 
oder der anderen Modification zu impfen, um das entsprechende Chlorhydrat zur Abscheidung zu bringen. Der Unterschied der unter verschiedenen Bedingungen, wie oben geschildert, zu erhaltenden gelben Verbindungen, der sich, wie schon erwähnt, in der Krystallform offenbart, findet auch in ihrer Zusammensetzung seinen Ausdruck.

Sämmtliche Verbindungen verlieren schon beim Stehen im Exsiccator Wasser und einen Theil der Salzsäure. Während die gelbe nadelförmige Verbindung $(A)$ beim Erhitzen auf $110^{\circ}$ fast vollständig weiss wird, nimmt die gelbe tafelförmige $(C)$ bei dieser Temperatur eine intensivere Gelbfärbung an.

Die weisse Modification wird beim Erhitzen theilweise gelb, so dass der Rückstand nicht homogen erscheint.

Sämmtliche Trockenrückstände. von welchen vorgreifend mitgetheilt werden soll, dass sie die Zusammensetzung

$$
\mathrm{C}_{20} \mathrm{H}_{20} \mathrm{~N}_{2} \mathrm{O}_{5} . \mathrm{HCl}
$$

haben, schmelzen unscharf zwischen $181-200^{\circ}$. Alle frisch bereiteten, zwischen Filtrirpapier getrockneten Chlorhydrate sind in Wasser, absolutem Alkohol leicht löslich (die gelben Salze schneller als die weissen), in Äther sind beide unlöslich, so dass dieselben aus concentrirter alkoholischer Lösung durch Äther ausgefällt werden können.

Die Trockenrückstände, gleichviel aus welchem Salze sie erhalten wurden, lösen sich nicht unzersetzt in Wasser, sie dissociiren unter Abscheidung von Oxim in gleicher Weise. Der Schmelzpunkt der so abgeschiedenen Oxime schwankt zwischen 245 und $248^{\circ}$.

Untersuchung des gelben nadelförmigen Chlorhydrates $(A)$.

Die Verbindung schmilzt bei $80-86^{\circ}$ im Krystallwasser, bei $109^{\circ}$ tritt Gasentwicklung ein, bei weiterem Erhitzen erstarrt die Substanz wieder, um wieder bei $210-230^{\circ}$ unter Gasentwicklung und Zersetzung zu schmelzen. Zur Analyse wurden die Substanzen, da sie alle, wie bereits erwähnt, schon beim Stehen an der Luft Salzsäure und Wasser abgeben, immer, auch die nachstehend beschriebenen, in gleicher Weise so lange zwischen Filtrirpapier gepresst und durch wiederholtes 
Zerkleinern der Substanz neue Oberflächen geschaffen, bis die Verbindung trocken $z u$ sein schien.

Von den nachstehenden Bestimmungen beziehen sich I, II und III auf Substanz von einer Darstellung, zu den übrigen wurde jedesmal Substanz von anderer Darstellung verwendet. Zur Wasserbestimmung wurde die zwischen Filtrirpapier getrocknete Substanz im Wasserstoffstrome im Luftbade aut $110^{\circ}$ bis zur Gewichtsconstanz erhitzt. Im vorgelegten Wasser wurde die Salzsäure titrimetrisch bestimmt und vom Gesammt. verlust abgezogen.

I. $0 \cdot 3012 \mathrm{~g}$ Substanz gaben $0 \cdot 4075 \mathrm{~g}$ Kohlendioxyd und $0 \cdot 1985 \mathrm{~g}$ Wasser.

II. $0 \cdot 2360 \mathrm{~g}$ Substanz gaben $0 \cdot 0963 g$ Chlorsilber.

III. $0 \cdot 5050 \mathrm{~g}$ Substanz bis zur Gewichtsconstanz auf $110^{\circ} \mathrm{im}$ Wasserstoffstrome erhitzt, verloren $0.1807 \mathrm{~g}$ an Gewicht, wovon auf Salzsäure $0.01825 \mathrm{~g}$ entfielen, welche durch Titration des rorgeschlagenen Wassers ermittelt wurden.

IV. $0.5289 \mathrm{~g}$ Substanz bis zur Gewichtsconstanz auf $110^{\circ}$ erhitzt, verloren $0 \cdot 1950 \mathrm{~g}$ an Gewicht, wovon auf Salzsäure $0.0182 \mathrm{~g}$ entfielen.

V. $0.7405 \mathrm{~g}$ Substanz bis zur Gewichtsconstanz auf $110^{\circ}$ erhitzt, verloren $0.2440 \mathrm{~g}$ an Gewicht, wovon $0.0230 \mathrm{~g}$ auf Salzsäure entfielen.

In 100 Theilen:

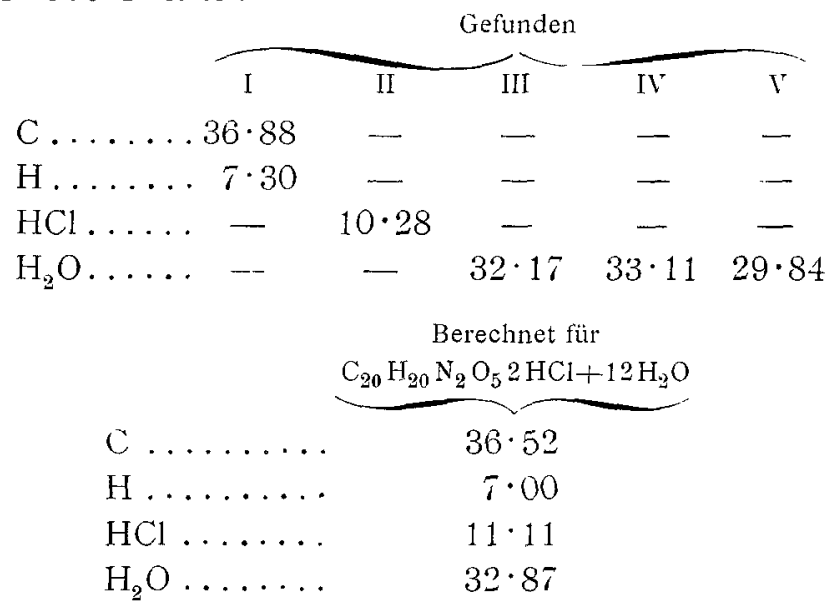


Aus diesen Analysen ist es wohl gestattet, den Schluss zu ziehen, dass das Chlorhydrat $A 2$ Moleküle Salzsäure und 12 Moleküle Krystallwasser enthält. Es ist demnach sowob1 an dem Stickstoff des Isochinolins, als auch an die Oximgruppe Salzsäure hinzugetreten.

Dass die Analysen insbesondere im Wasser und Salzsäuregehalt nicht besonders gut mit den theoretischen Werthen übereinstimmen und auch untereinander etwas grosse Differenzen zeigen, ist dem bereits erörterten Umstande zuzuschreiben, dass es kaum möglich ist, lufttrockene, aber nicht vervitterte Substanz zur Analyse zu bringen. Überdies kommt hier vielleicht auch noch in Betracht, dass dem Salze wahrscheinlich, unter Umständen, auch solches von geringerem Wassergehalte in kleiner Menge beigemengt sein könnte.

Die Analysen der fast rein weissen Trockenrückstände obiger Salze ergaben folgende Resultate:

1. $0 \cdot 2043 g$ Substanz gaben $0 \cdot 4450 \mathrm{~g}$ Kohlendioxyd und $0.0998 g$ Wasser.

II. $0 \cdot 3130 \mathrm{~g}$ Substanz gaben $0 \cdot 1069 \mathrm{~g}$ Chlorsilber.

III. $0 \cdot 2925 g$ Substanz gaben $0 \cdot 0961 g$ Chlorsilber.

IV. $0 \cdot 2892 \mathrm{~g}$ Substanz gaben $17.6 \mathrm{~cm}^{3}$ feuchten Stickstoft bei $14.5^{\circ} \mathrm{C}$ und $737 \mathrm{~mm}$ Barometerstand.

In 100 Theilen:

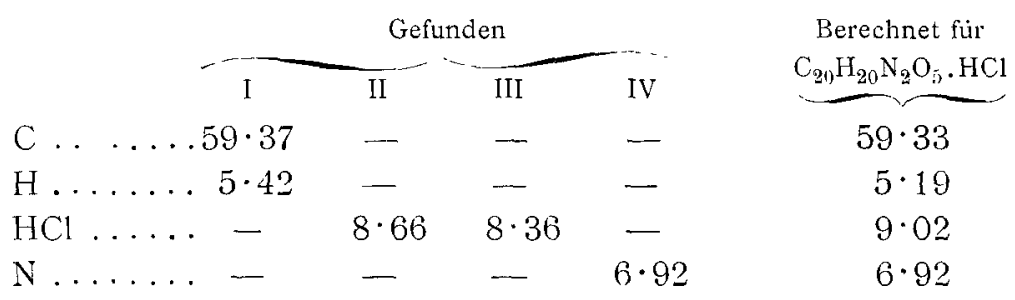

Der weisse Trockenrückstand obigen Salzes ist demnach wasserfreies Oximchlorhydrat von der empirischen Formel $\mathrm{C}_{20} \mathrm{~N}_{20} \mathrm{H}_{2} \mathrm{O}_{5} \mathrm{HCl}$. Da Papaveraldinchlorhydrat beim Erhitzen auf 
$110^{\circ}$ schon leicht sämmtliche Salzsäure verliert, so dürfte der Substanz wohl nachstehende rationelle Formel

$$
\begin{gathered}
\left(\mathrm{CH}_{3} \mathrm{O}\right)_{2} \mathrm{C}_{6} \mathrm{H}_{3} \mathrm{C}-\mathrm{C}_{9} \mathrm{H}_{4} \mathrm{~N}\left(\mathrm{OCH}_{3}\right)_{2} \\
\mathbb{N ~ O H ~ H C l}
\end{gathered}
$$

zukommen.

Dieser Rückstand beginnt bei $195^{\circ} \mathrm{zu}$ schmelzen, weiter erhitzt tritt bei $210^{\circ}$ Braunfärbung ein.

\section{Untersuchung des gelben, in rhombischen Tafeln krystallisi- renden Oximchlorhydrats $(C)$.}

Die Verbindung schmilzt bei $95^{\circ}$, bei $110^{\circ}$ tritt Gasentwicklung unter starker Gelbfärbung ein; noch weiter erhitzt, wird die geschmolzene Masse bei $210^{\circ}$ unter Braunfärbung zersetzt. Auch hier musste wegen der Schwierigkeit trockene und anderseits unverwitterte Substanz zur Analyse zu bringen, wie bei den Analysen von $A$ die abfiltrirte Substanz, nachdem sie scharf $z$ wischen Filtrirpapier gepresst worden war, direct verwendet werden.

Die Bestimmungen I, III, $\mathrm{V}$ beziehen sich auf Substanz derselben Darstellung, II, IV auf solche einer zweiten und VI auf Substanz einer dritten Bereitung.

I. $0 \cdot 2910 \mathrm{~g}$ Substanz gaben $0 \cdot 5065 \mathrm{~g}$ Kohlendioxyd und $0 \cdot 1490 \mathrm{~g}$ Wasser.

II. $0 \cdot 2427 \mathrm{~g}$ Substanz gaben $11 \mathrm{~cm}^{3}$ feuchten Stickstoff bei $16 \cdot 5^{\circ} \mathrm{C}$. und $751.6 \mathrm{~mm}$ Barometerstand.

III. $0 \cdot 2580 \mathrm{~g}$ Substanz gaben $0 \cdot 1365 \mathrm{~g}$ Chlorsilber.

IV. $0 \cdot 3031 \mathrm{~g}$ Substanz gaben $0 \cdot 1615 \mathrm{~g}$ Chlorsilber.

V. $0 \cdot 3360 \mathrm{~g}$ Substanz verloren bis zur Gewichtsconstanz auf $110^{\circ}$ erhitzt $0.655 \mathrm{~g}$ an Gewicht, wovon auf Salzsäure $0.0182 g$ entfielen.

VI. $0.4113 \mathrm{~g}$ Substanz bis zur Gewichtsconstanz auf $110^{\circ}$ getrocknet, verloren $0.0906 \mathrm{~g}$ an Gewicht, wovon auf Salzsäure $0.0255 \mathrm{~g}$ entfielen. 
In 100 Theilen:

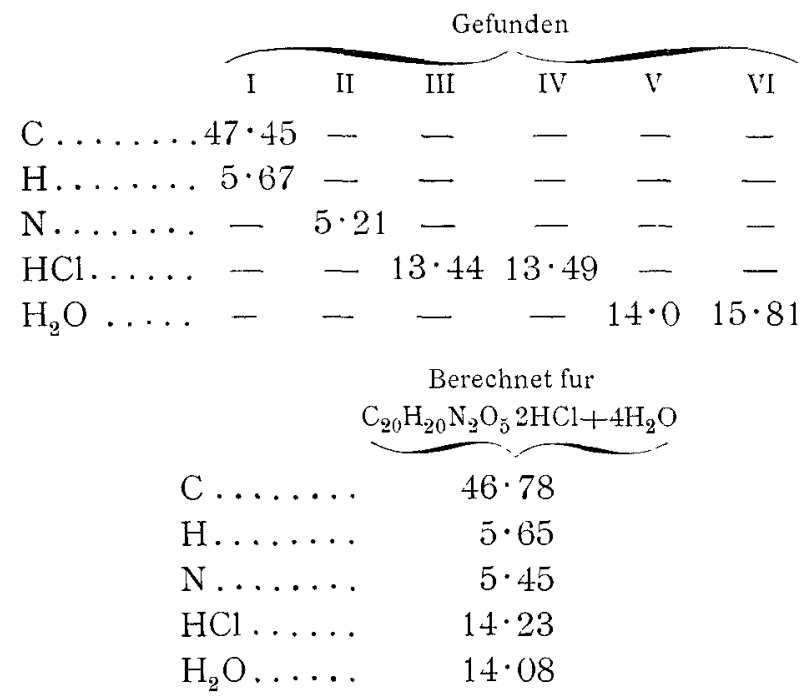

Aus diesen Analysen, welche, wie zu erwarten war, aus den bereits hervorgehobenen Gründen keine scharf stimmenden Zahlen ergaben, ist zu entnehmen, dass dieses Salz, das immer unter den oben angegebenen Versuchsbedingungen entsteht, sich von dem erstbeschriebenen $(A)$ durch den Krystallwassergehalt unterscheidet. Bemerkenswerth ist, dass beim Trocknen des Salzes bei $110^{\circ}$, also bei derselben Temperatur, welche auch bei $A$ eingehalten wurde, Rückstände erhalten wurden, welche nach den untenstehenden analytischen Bestimmungen dieselbe Zusammensetzung wie jene von $A$ haben, aber intensiv gelb gefärbt sind, während jene weiss wurden.

Diese gelben Rückstände schmelzen bei $170^{\circ}$, bei $180^{\circ}$ tritt Gasentwicklung und Dunkelfärbung ein.

I. $0 \cdot 3169 \mathrm{~g}$ Substanz gaben $0 \cdot 1090 \mathrm{~g}$ Chlorsilber.

II. $0.2592 \mathrm{~g}$ Substanz gaben $0.0889 \mathrm{~g}$ Chlorsilber.

In 100 Theilen:

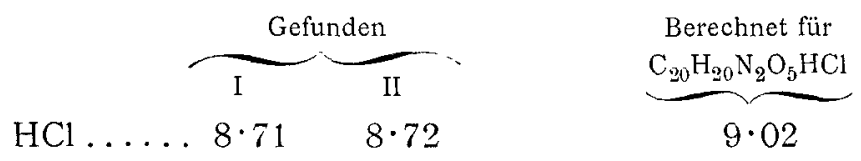




\section{Untersuchung des Papaveraldoximchlorhydrats $(D)$.}

Das ebenfalls gelbgefärbte Chlorhydrat war entstanden, als Papaveraldoxim vom Schmelzpunkt $238^{\circ}$ mit wässeriger $7 \%$ Salzsäure 5 Stunden am Rückflusskühler gekocht worden war. Es stellte kleine nadelförmige Krystalle dar, die äusserlich dem Chlorhydrate $A$ sehr ähnlich waren, die aber, wie die Analyse zeigt, einen bedeutend geringeren Salzsäure- und Wassergehalt hatten.

I. $0 \cdot 2460 \mathrm{~g}$ Substanz gaben $0 \cdot 4700 \mathrm{~g}$ Kohlendioxyd und $0 \cdot 1298 \mathrm{~g}$ Wasser.

II. $0 \cdot 2043 g$ Substanz auf $130^{\circ}$ bis zur Gewichtsconstanz erhitzt, verloren $0.0316 g$ an Gewicht, wovon auf Salzsäure $0.0089 g$ entfielen.

In 100 Thellen:

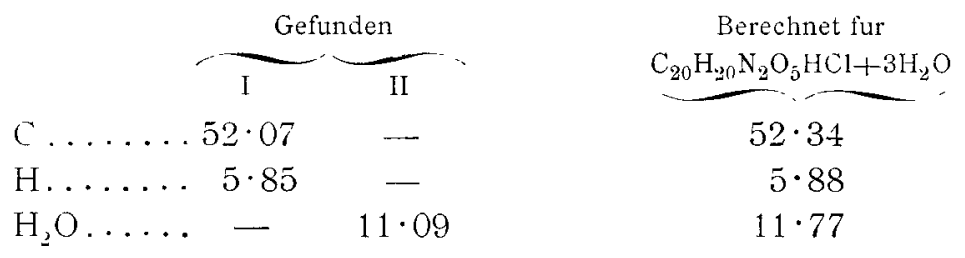

$0 \cdot 1727 g$ überwiegend gelber Trockenrückstand gaben $0.0433 \mathrm{~g}$ Chlorsilber.

In 100 Theilen:

$$
\mathrm{HCl} \ldots \ldots \underbrace{\text { Gefunden }}_{6 \cdot 36} \quad \frac{\begin{array}{c}
\text { Berechnet für } \\
\mathrm{C}_{20} \mathrm{H}_{20} \mathrm{~N}_{2} \mathrm{O}_{5} \cdot \mathrm{HCl}
\end{array}}{9 \cdot 02}
$$

Die grosse Differenz im Chlorgehalte dürfte im folgenden senne Erklärung finden:

Da das Chlorhydrat beim Erhitzen auf $110^{\circ}$ an der der Flamme zugewendeten Seite des die Substanz enthaltenden Schiffchens weiss geworden war, wurde versucht, ob durch stärkeres Erhitzen die Substanz ganz weiss zu erhalten wäre, was selbst bei $130^{\circ}$ nicht erreicht wurde; hiebei hatte sich ein weiterer Gewichtsverlust ergeben. 
Rechnet man nun zu dem, direct im Rückstande gefundenen Salzsäuregehalt, jenen hinzu, welcher beim Erhitzen der wasserhältigen Substanz entwichen und durch Titration des vorgeschlagenen Wassers ermittelt worden war, so ergibt sich, auf trockene Substanz bezogen, nachstehender Gesammtchlorgehalt.

Die bei Analyse II gewogene Substanz $=0.2043 g$ lieferte im Wasserstoffstrom erhitzt $0.00894 \mathrm{~g}$ Chlorwasserstoffsäure, im Rückstande $0.0110 \mathrm{~g}$ Salzsäure, daher im Ganzen $0.0199 g$, welche, auf Rückstand plus beim Erhitzen entwichene Salzsäure bezogen, ergibt:

In 100 Theilen:

$$
\begin{aligned}
& \text { Gefunden } \\
& 10 \cdot 97 \\
& \mathrm{FCl} \ldots \ldots \quad 10 \cdot 97
\end{aligned}
$$

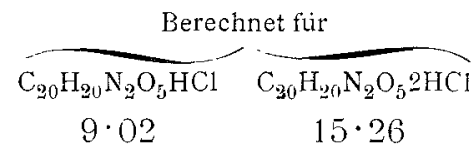

Mit Rücksicht auf die geringe Menge Substanz, welche fur die Chlorbestimmung noch zur Verfügung stand, auf die Ungenauigkeit, welche der Titration bei so kleinen Quantitäten anhaftet, auf die schon wiederholt erwähnten, in der Natur dieser Verbindungen gelegenen Schwierigkeiten bei der Abwägung derselben, ist man wohl auf Grund der Analysen trotz der nicht befriedigenden Übereinstimmung im Chlorgehalte anzunehmen berechtigt, dass unter den eingehaltenen Versuchsbedingungen ein Salz von der Zusammensetzung $\mathrm{C}_{20} \mathrm{H}_{30} \mathrm{~N}_{2} \mathrm{O}_{5} \cdot \mathrm{HCl}+3 \mathrm{H}_{2} \mathrm{O}$ entstehe.

\section{Untersuchung des weissen Oximchlorhydrates $(B)$.}

Diese in Bezug auf ihre Entstehungsbedingungen und auf ihr Aussehen bereits beschriebene Substanz sintert bei $90^{\circ}$ und schmilzt bei $220^{\circ}-225^{\circ}$ unter Gelbfärbung und Gasentwicklung. Es wurde festgestellt, dass die Substanz im Exsiccator bis zur Gewichtsconstanz getrocknet $18 \cdot 56 \%$ an Gewicht verliert und dass dabei nicht nur Wasser, sondern auch Salzsäure fortgeht. 
I. $0.3050 \mathrm{~g}$ zwischen Filtrirpapier getrocknete Substanz gaben $0.4558 \mathrm{~g}$ Kohlendioxyd und $0.1930 \mathrm{~g}$ Wasser.

II. $0.6073 \mathrm{~g}$ zwischen Filtrirpapier getrocknete Substanz. verloren beim Erhitzen auf $110^{\circ}$ bis zur Gewichtsconstanz $0.1988 \mathrm{~g}$ an Gewicht, wovon auf Salzsäure $0.0113 \mathrm{~g}$ entfielen.

III. $0.2778 g$ Substanz, zwischen Filtrirpapier getrocknet, verloren bis zur Gewichtsconstanz auf $110^{\circ}$ erhitzt $0.0915 \mathrm{~g}$ an Gewicht, wovon $0.0058 \mathrm{~g}$ auf Salzsäure entfielen.

IV. $0 \cdot 3432 g$ zwischen Filtrirpapier getrocknete Substanz gaben $0 \cdot 1173 \mathrm{~g}$ Chlorsilber.

V. $0 \cdot 2443 \mathrm{~g}$ zwischen Filtrirpapier getrocknete Substanz gaben $0.0819 \mathrm{~g}$ Chlorsilber.

VI. $0 \cdot 3162 g$ zwrischen Filtrirpapier getrocknete Substanz gaben $0 \cdot 1316 g$ Chlorsilber.

In 100 Theilen:

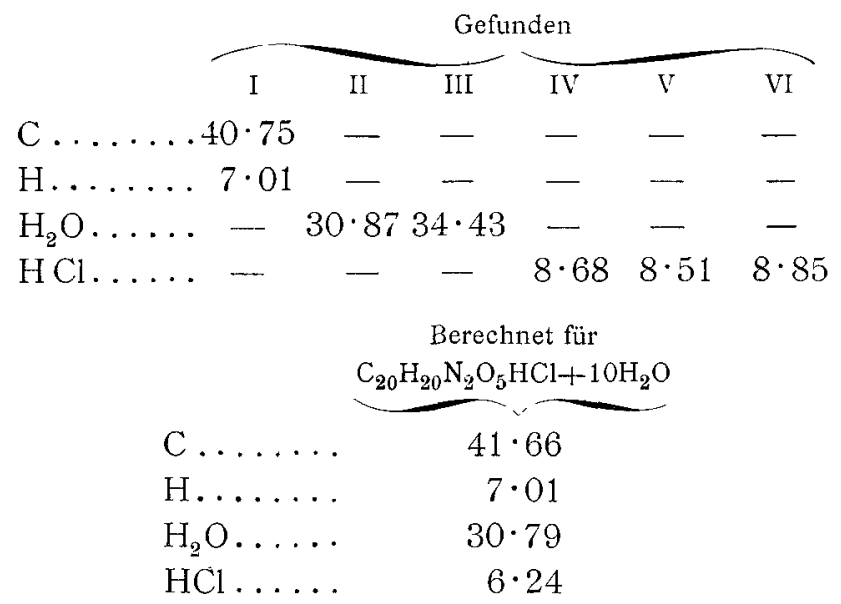

Von diesen Bestimmungen stammen I, II und IV von Substanz derselben Darstellung, während III, V und VI von drei anderen Bereitungen stammen. Es scheint also, dass dieses weisse Chlorhydrat nur 1 Molekül Salzsäure und 10 Moleküle Krystallwasser enthalte; möglicherweise ist demselben eine kleine Menge einer Verbindung, die 2 Moleküle Salzsäure enthält, beigemengt oder haftet ihnen etwas freie Salzsäure an. 
Die Analysen der gelblich-iweissen Trockenrückstände, die bei $185-187^{\circ}$ schmelzen, ergaben folgende Resultate:

I. $0 \cdot 1695 g$ Substanz gaben $0 \cdot 3682 g$ Kohlendioxyd und $0.0841 \mathrm{~g}$ Wasser.

II. $0.2250 \mathrm{~g}$ Substanz gaben $0.0765 g$ Chlorsilber.

III. $0 \cdot 1858 \mathrm{~g}$ Substanz gaben $0 \cdot 0655 \mathrm{~g}$ Chlorsilber.

In 100 Theilen:

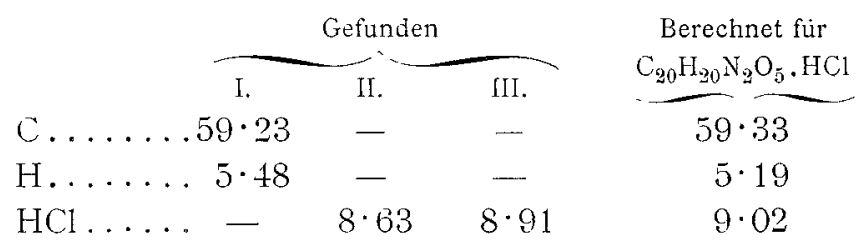

Dieser $\mathrm{schwach}$ gelbliche Trockenrückstand des we issen Chlorhydrat $\mathrm{C}_{20} \mathrm{H}_{20} \mathrm{~N}_{2} \mathrm{O}_{5} \mathrm{HCl}+3 \mathrm{H}_{2} \mathrm{O}(B)$ hat demnach dieselbe Zusammensetzung wie der weisse Trockenrückstand des gelben Salzes $\mathrm{C}_{20} \mathrm{H}_{20} \mathrm{~N}_{2} \mathrm{O}_{5} 2 \mathrm{HCl}+12 \mathrm{H}_{2} \mathrm{O}(A)$ und wie der gelbe Rückstand des ebenfalls gelben Salzes $\mathrm{C}_{20} \mathrm{H}_{20} \mathrm{~N}_{2} \mathrm{O}_{5} 2 \mathrm{HCl}+4 \mathrm{H}_{2} \mathrm{O}(C)$. Die Rückstände unterscheiden sich auch durch ihre Schmelzpunkte, und zwar liegt derjenige des schwach gelblich gefärbten, aus dem weissen Salze $B$ erhaltene bei $185-187^{\circ}$, während der gelbe Rückstand aus $C$ bei $170^{\circ}$, der weisse aus $A$ bei $195-210^{\circ}$ beobachtet wurde. Vielleicht ist, darauf deutet Färbung und Schmelzpunkt hin, der Rückstand von $C$ ein Gemenge jener von $A$ und $B$.

\section{Versuch der Configurationsbestimmung des Papaveraldoxims vom Schmelzpunkte $235^{\circ}$.}

I. Umlagerungsversuch mit Phosphorpentachlorid nach der von $\mathrm{Hantzsch}{ }^{1}$ angegebenen Methode.

$2 \mathrm{~g}$ fein gepulvertes Oxim wurden in $100 \mathrm{~cm}^{3}$ absolut trockenen Äthers suspendirt und unter Kühlung und öfterem Schütteln Phosphorpentachlorid in kleinen Portionen so lange

1 Berl. Ber., XXIV, S. 13. 
hinzugesetzt, bis ein grösserer Überschuss am Boden des Gefässes sichtbar wurde $(3 \mathrm{~g})$. Die Einwirkung des Phosphorpentachlorids begann alsbald, was an der Gelbfärbung des Äthers, wie auch an der des aufgeschlemmten Oxims ersichtlich war. Zur rascheren Beendigung dieser Reaction wurde das Reactionsgemisch in einem Schüttelapparate 48 Stunden hindurch in fortwährender Bewegung erhalten. Das ungelöst gebliebene Product war nach dieser Zeit intensiv roth, der Äther schwach gelblich gefärbt. Der Äther hinterliess nach dem Destilliren am Wasserbade einen Rückstand, der stark nach Phosphoroxychlorid und Salzsäure roch. Das lufttrockene rothe, wie Mennige aussehende Reactionsproduct, dem noch Phosphorpentachlorid beigemengt war, wurde nun tropfenweise mit destillirtem Wasser versetzt, wobei Salzsäureentwicklung stattfand. Das Product nahm eine blutrothe Färbung an, ging theilweise in Lösung, fiel jedoch bei grösserem Wasserzusatz wieder als orangerother Niederschlag aus, der abgesaugt, gewaschen und auf Thon getrocknet wurde. Dieser Körper färbte sich bei $170^{\circ}$ hellgelb, bei $215^{\circ}$ weiss und schmolz bei $238^{\circ}$ unter starker Gasentwicklung und gleichzeitiger Zersetzung. Er löste sich in absolutem Alkohol beim Kochen mit orangerother Farbe und krystallisirte nach dem Erkalten in gelben Nadeln aus, welche sich bei $238^{\circ}$ dunkel färben und bei $244^{\circ}$ unter Zersetzung schmelzen. Die Verbindung enthält Chlor, welches, sowohl durch Kochen mit Wasser, als durch Verreiben mit Natronlauge, der Verbindung entzogen werden kann. Zur quantitativen Chlorbestimmung dieser Verbindung wurde mit chlorfreier Natronlauge dreimal gekocht und die Salzsäure im Filtrat mit Silbernitrat gefällt.

Nachstehende Analyse zeigt, dass die Substanz, welche gewiss verschieden von diesen, denselben Chlorgehalt hat, wie die Trockenrïckstände der Oximchlorhydrate; dies mụsste erwartet werden, wenn die Beckmann'sche Umlagerung stattgefunden hatte; das entstandene Umlagerungsproduct musste noch die Fähigkeit haben, am Isochinolinstickstoff Salzsäure zu fixiren.

$0 \cdot 5000 g$ Substanz gaben 0.1687 g Silberchlorid. 
In 100 Theilen:

$$
\mathrm{HCl} \ldots \ldots \underbrace{\text { Gefunden }}_{8 \cdot 54} \quad \frac{\begin{array}{c}
\text { Berechnet für } \\
\mathrm{C}_{20} \mathrm{H}_{20} \mathrm{~N}_{2} \mathrm{O}_{5} \mathrm{HCl}
\end{array}}{9 \cdot 02}
$$

Der Schmelzpunkt der durch Natronlauge abgeschiedenen schwach gelb gefärbten Substanz liegt bei $170^{\circ}$.

Die Verbrennung ergab Zahlen, wie sie den durch die Beckmann'sche Umlagerung zu erwartenden, dem Oxime isomeren Körpern

I.

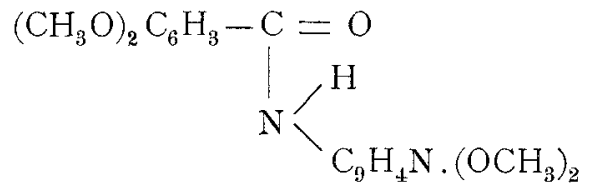

Veratrumsäure-B-2, 3-Dimethoxylisochinolylamid

oder

II.<smiles>CCCCNC(=O)NCCCCCOC</smiles>

$B-2,3$-Dimethoxylisochinolincarbonsäure-Veratrylamid

zukommen mussten.

$0.1726 \mathrm{~g}$ Substanz gaben $0.4115 \mathrm{~g}$ Kohlendioxyd und $0.0825 \mathrm{~g}$ Wasser.

In 100 Theilen:
Gefunden
C...... $65 \cdot 0$
H...... $5 \cdot 31$
$\begin{array}{r}\text { Berechnet für } \\ \mathrm{C}_{20} \mathrm{H}_{20} \mathrm{~N}_{2} \mathrm{O}_{5} \\ \hline\end{array}$
$65 \cdot 21$
$5 \cdot 43$

Um zu erfahren, welche der beiden Formeln dem vorliegenden Körper entspricht und hiedurch die Configuration des niedrig schmelzenden Oxims festzustellen, wurde die Substanz mit concentrirter Salzsäure vom spec. Gew. $1 \cdot 15$ vier Stunden 
im geschlossenen Rohr auf $160^{\circ}$ erhitzt, um die hydrolytische Spaltung herbeizuführen. Der Inhalt war dunkel gefärbt und bis auf einen geringen schwarzen Rest war Alles in Lösung gegangen. Im Rohr war starker Druck vorhanden, das entweichende Gas färbte die Gasflamme grün (Chlormethyl); in Barytwasser geleitet, entstand Trübung.

Die stark saure Reactionsflüssigkeit wurde mit Wasser verdünnt und in Kohlensäureatmosphäre mit Äther bis zur Erschöpfung behandelt. Die röthlich gefärbten ätherischen Auszüge wurden vereinigt und der $\ddot{A}$ ther am Wasserbade abdestillirt, der schmierigbraune Rückstand wurde mit Wasser in einer Kohlensäureatmosphäre gekocht, von unlöslichen schwarzen Flocken filtrirt, das Filtrat mit Äther abermals ausgeschüttelt, dieser dann im Vacuum eingedunstet, wobei gelblich gefärbte, krystallinische Nadeln in geringer Menge zurückblieben.

Diese Nadeln sind in Wasser leicht löslich und wurden durch nachstehende Reactionen als mit Brenzcatechin identisch erkannt.

1. Eisenchlorid färbt die Lösung smaragdgrün, bei Zusatz von Ammoniak schlägt die Färbung in Roth über.

2. Ammoniakalische Silbernitratlösung wird in der Kälte reducirt.

3. Fehling'sche Lösung wird erst beim Kochen reducirt.

4. Bleizucker erzeugt keinen Niederschlag.

5 . Bleiessig erzeugt einen weissen Niederschlag.

Dieser Befund bestätigte die schon a priori naheliegende Vermuthung, die durch die Beobachtungen beim Öffnen der Druckröhren bestätigt wurde, dass die Salzsäure bei der Versuchstemperatur nicht nur hydrolytische Spaltung des Umlagerungsproductes, sondern auch Abspaltung der Methoxylgruppen bewirken werde.

Es musste daher aus dem der Formel I entsprechenden Veratrumsäure-B-2-, 3-Dimethoxylisochinolinamid, Veratrumsäure und dann aus dieser Protocatechusäure, schliesslich Brenzcatechin gebildet worden sein. Ein besonderer Versuch zeigte, dass Protocatechusäure bei $160^{\circ}$ mit Salzsäure derselben Concentration wie die angewendete, in der That in Kohlendioxyd und Brenzcatechin zersetzt werde. Leider konnte aus 
der Reactionsmasse weder aus saurer, noch alkalischer Lösung sonst ein brauchbares Product isolirt werden, da in Folge der Abspaltung von Methyl der Methoxylgruppen, hydroxylhältige Spaltungsproducte entstanden waren, die selbst in sehr verdünnter Lösung sehr intensive Farbenreactionen mit Eisenchlorid gaben, aber nahezu schwarz gefärht waren und absolut keine Neigung zur Krystallisation zeigten.

Mit Rücksicht auf die nur geringe Menge Brenzcatechin, welche nachgewiesen werden konnte, ist ein sicherer Schluss auf die Configuration des in Arbeit genommenen Oxims nicht zulässig, da ja möglicherweise auch alle vier Spaltungsproducte. welche hier in Betracht kommen, entstanden sein können und drei hievon sich in Folge der Nebenwirkung, welche die Salzsäure ausübt, der Beobachtung entzogen haben konnten.

Ähnlichen Erfolg hatte auch ein Versuch durch Anwendung ron Beckmann'scher Mischung (mit Salzsäure gesättigter Eisessig und Essigsäureanhydrid) in der Hitze, die Configuration des Oxims zu bestimmen. Auch hier konnte nur Brenzcatechin nachgewiesen werden. Bei der Behandlung des Oxims (Schmelzpunkt $235^{\circ}$ ) mit Schwefelsäure, die mit einem Drittel ihres Gewichtes Wasser verdünnt worden war, wurde nach 3 Minuten langem Erwärmen im Wasserbade im Filtrate der verdunnten Lösung, aus welcher sich ein gelbes Sulfat - wahrscheinlich saures Papaveraldinsulfat - ausgeschieden hatte, durch Natriumcarbonat noch unverändertes Oxim nachgerriesen. Dasselbe wurde durch das typische Verhalten - Umwandlung seines gelben Chlorhydrates durch Rühren mit einem Glasstabe in das weisse - erkannt. Der Versuch wurde daher wiederholt, jedoch 10 Minuten lang am Wasserbade erhitzt. Jetzt konnte nur mehr Papaveraldin aus der Lösung isolirt werden.

\section{Papaveraldylamin.}

Aldoxime wie Ketoxime geben, wie dies zuerst H. Goldschmidt ${ }^{1}$ nachgewiesen hat, bei der Reduction primäre Amine.

Es ist mir gelungen, auch das Papaveraldoxim in saurer Lösung mit Natriumamalgam zu reduciren und ich gelangte so zu einer Base, dem Papaveraldylamin, die ein grösseres

1 Berichte der Deutschen chem. Gesellschaft, XIX, 3232. 
Interesse verdient, weil durch die Reduction das Kohlenstoffatom der Carbonylgruppe assymetrisch wird.

Die neue Base muss nach folgender Gleichung entstanden sein:
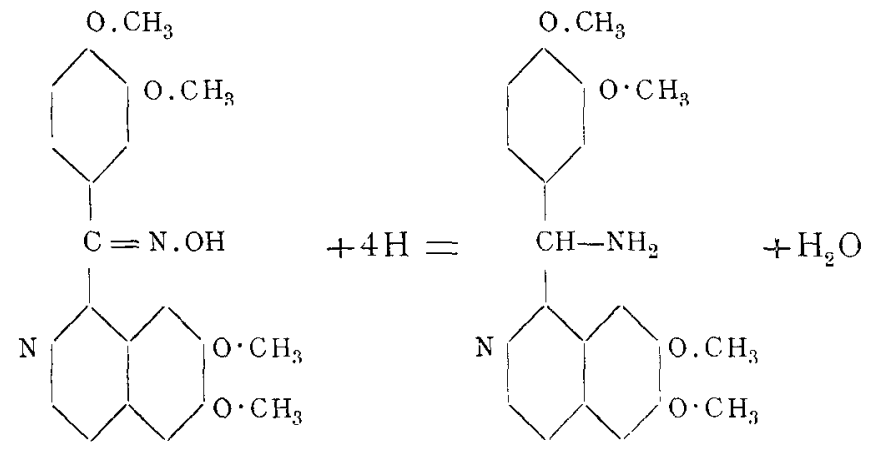

$2 \mathrm{~g}$ Oxim rom Schmelzpunkte $235^{\circ}$ wurden in $300 \mathrm{~cm}^{3}$ absoluten Alkohol gelöst und in die klare, farblose, abgekühite Lösung, die durch successiven Zusatz von Eisessig stets sauer erhalten wurde, bei gewöhnlicher Temperatur nach und nach 200 g $2^{1} / 2$ procentiges Natriumamalgam eingetragen. Die Reduction dauerte 5 Stunden. Die Reactionsflüssigkeit war schwach gelblich gefärbt und gegen Ende der Reaction konnte eine Erwärmung auf $35^{\circ}$ constatirt werden. Die vom Quecksilber und vom ausgeschiedenen essigsauren Natron befreite alkoholische Lösung wurde auf ein Drittel des Volumens eingeengt und in die dreifache Menge Wasser gegossen. Dann wurde mit kohlensaurem Natron bis zur alkalischen Reaction versetzt, wobei sich ein gelber Körper, theils schmierig, theils flockig abschied, der beim Schütteln mit Äther in diesen überging. Der ätherische Auszug wurde über calcinirter Pottasche getrocknet und ein Theil mit trockenem Salzsäuregas behandelt. Es fiel ein gelblich weisser voluminöser Niederschlag aus, der abfiltrirt und im Vacuum getrocknet, analysirt wurde.

$0 \cdot 4980 \mathrm{~g}$ Substanz gaben $0 \cdot 1910 \mathrm{~g}$ Chlorsilber.

In 100 Theilen:

\begin{tabular}{|c|c|c|}
\hline & Gefunden & $\begin{array}{l}\text { Berechnet für } \\
\mathrm{C}_{20} \mathrm{H}_{22} \mathrm{~N}_{2} \mathrm{O}_{4} \mathrm{HCl}\end{array}$ \\
\hline $\mathrm{HCl} \ldots \ldots$ & $9 \cdot 70$ & $9 \cdot 34$ \\
\hline
\end{tabular}


Das Chlorhydrat ist in Wasser und absolutem Alkohol leicht löslich.

Um die freie Base zu gewinnen, wurde der Äther vom anderen Theile der Lösung am Wasserbade abdestillirt, wobei ein gelbbraunes, durchsichtiges, vanillenartig riechendes Öl zurückblieb, das im Luftpumpenexsiccator zu einer durchsichtigen, gelbbraunen Masse erstarrte. Der Körper ist in absolutem Alkohol und Äther leicht löslich und schmilzt bei $80-85^{\circ}$ unter Aufschäumen.

Nachfolgende Kohlen- und Wasserstoffbestimmung zeigt, dass thatsächlich die freie Base vorlag.

$0 \cdot 1490 \mathrm{~g}$ Substanz gaben $0.3720 \mathrm{~g}$ Kohlendioxyd und $0.0890 \mathrm{~g}$ Wasser.

In 100 Theilen:

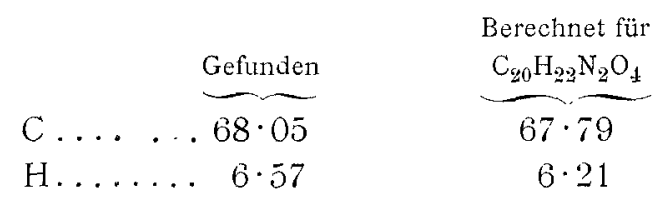

Die salzsaure Lösung der Base gab mit Platinchlorid sofort einen gelben flockigen Niederschlag, der mit Rücksicht auf die geringe Menge, die zur Verfügung stand, nicht analysirt werden konnte. 